\title{
The Role of the Pharmacist in the Care of Patients with Chronic Pain
}

\author{
Laura Murphy' \\ Karen $\mathrm{Ng}^{2}$ \\ Pearl Isaac ${ }^{3}$ \\ Jaris Swidrovich (iD) 4 \\ Maria Zhang ${ }^{3,5}$ \\ Beth A Sproule 3,5 \\ 'Department of Pharmacy, University \\ Health Network, Toronto, ON, Canada; \\ ${ }^{2}$ Toronto Academic Pain Medicine \\ Institute, Toronto, ON, Canada; \\ ${ }^{3}$ Pharmacy Department, Centre for \\ Addiction and Mental Health, Toronto, \\ ON, Canada; ${ }^{4}$ College of Pharmacy and \\ Nutrition, University of Saskatchewan, \\ Saskatoon, SK, Canada; ${ }^{5}$ Leslie Dan \\ Faculty of Pharmacy, University of \\ Toronto, Toronto, ON, Canada
}

\begin{abstract}
Pharmacists across the healthcare continuum are well positioned to collaborate with patients to effectively manage their chronic pain. Evidence supports positive outcomes when pharmacists undertake these roles; however, there are barriers preventing uptake across the profession. This paper aims to expand awareness of the breadth of these roles, including pharmaceutical care provision, interprofessional collaboration, pain and medication education, support for patients in self-management and acceptance of responsibility to be culturally responsive and decrease stigma. Pharmacists are accessible healthcare professionals and can improve the care of patients with chronic pain.
\end{abstract}

Keywords: chronic pain, pharmacist, patient-centered care, stigma

\section{Introduction}

Chronic pain is a significant health condition affecting one in five people globally. ${ }^{1}$ It is associated with a complex interplay of physical, emotional, mental and spiritual challenges that influence each other and result in poor function, reduced quality of life and stigma. ${ }^{2,3}$ Adverse childhood experiences and traumatic events can worsen patients' pain experiences and morbidity. ${ }^{4}$ Pharmacotherapy is only one modality for pain management; physical, psychological, spiritual, and other treatment approaches also have important roles. Pharmacists and other members of the interprofessional team (eg, physicians, nurses, occupational therapists, physical therapists, psychologists, others) must collaborate to achieve positive patient outcomes. ${ }^{5-7}$ Pharmacists are accessible members of the team who partner with patients to optimize safe use of medications for chronic pain and comorbidities. They are also well positioned to support pain self-management and pain education, collaborate with other health and wellness care providers, and reduce stigma experienced by the patient. With a patient-centered, trauma-informed approach to care, pharmacists can improve patient experiences and outcomes. ${ }^{8}$ This paper aims to expand on the evidence for pharmacist's roles in managing chronic pain described in the literature, drawing on our collective experience caring for patients with chronic pain within specialty ambulatory clinics, inpatient hospital and rehabilitation settings, and community pharmacies across Canada. This is not a formal knowledge synthesis paper. Patient vignettes drawn from our clinical experiences are included to illustrate opportunities for pharmacists' roles in the care of patients with chronic pain.
Correspondence: Beth A Sproule Centre for Addiction and Mental Health (CAMH), 1025 Queen Street West, Toronto, ON, M6J IH4, Canada

Tel + I-4I6-535-850I x3560I

Fax $+1-416-583-1277$

Email beth.sproule@camh.ca 


\section{Evidence for the Role of the Pharmacist in the Management of Chronic Pain}

To contribute to the narrative on the topic of the role of the pharmacist in the management of chronic pain, a scan of the literature was conducted using the keywords "pharmacist" and "chronic pain". Our approach was to first highlight the most robust evidence available; a systematic review and meta-analyses published in 2021. ${ }^{9}$ Thapa et al included studies in their review if they involved pharmacist-delivered intervention either alone or within a multidisciplinary team (medication review, pharmaceutical care, patient education or counselling) to patients with chronic noncancer pain, excluding review articles and conference poster abstracts. Fourteen studies were included in the review, with a total of 2365 participants from six randomized controlled trials (RCT), and nine observational studies. Medication review was the most common intervention provided by the pharmacist. Pharmacist-led interventions reduced pain intensity for patients with chronic pain (outcome reported in five RCTs), and these were more effective if they occurred over at least 3 months. Interventions had minimal effect on physical functioning (outcome reported in five RCTs). Pooled results from observational studies for these outcomes had mixed effects. Pharmacists conducted opioid stewardship in three observational studies resulting in effective dose reduction. Only one study examined costs, and determined that pharmacist intervention (costs of training, intervention delivery and follow-up, medication, and primary and secondary care activities) was more expensive than treatment as usual.

In addition to this meta-analysis, our scan of the literature identified other descriptive papers of interest to this topic. Dole et al described a pharmacist-led chronic pain and opioid management clinic in 2007 where pharmacists had delegated prescribing authority to act independently. Data from 1 year pointed to improved pain outcomes and significant cost-savings to the healthcare system. ${ }^{10}$ Wiedemer et al described a pharmacist's role within the team primarily focused on opioid therapy management, and reported improved adherence to guidelines and reduction in aberrant behaviours over a two 2 year period. ${ }^{11}$

Other papers described pharmacist-led opioid or nonsteroidal anti-inflammatory drug (NSAID) specific risk assessment services and screening initiatives for patients with chronic pain in community pharmacies and primary care practices leading to positive impacts when pharmacists undertook this role in care. ${ }^{12-14}$

Despite a breadth of pharmacist-led practices for chronic pain and related positive outcomes in the literature, uptake of these roles across the profession has been limited. Studies have highlighted that this may be due to a lack of clarity around the responsibilities of the pharmacist and their perceived scope of practice and a need for institutional support (time, staffing, training) in some practice settings. $^{15-18}$

\section{Role in Medication Management}

Chronic pain is often accompanied by comorbidities related to mood and sleep, substance use and use disorders, and other chronic conditions. If insufficiently managed, these comorbidities can have a significant impact on the pain experience. They can also result in complex medication regimens that can increase risks of drug interactions and side effects.

\section{Pharmaceutical Care}

- Mr. S is 78 years old, he has a 20-year history of chronic mechanical low back pain with neuropathic features, sleep disturbances, and anxiety. He also has mild cognitive impairment, benign prostatic hypertrophy, and renal dysfunction. Treatment options for surgery and injections have been ruled out by his doctor. He was recently prescribed oxycodone, but is having minimal relief. He frequently asks his physician and pharmacist for more pills, and "something stronger". He recently went to his local Emergency room with the same request. His family has gathered other medications prescribed to him, including several prescriptions for benzodiazepines, over the counter sleeping medications, baclofen, and citalopram, which he started last month for his anxiety.

\section{Gathering a Best Possible Medication History}

The best possible medication history (BPMH) should include drug allergies and all current prescription and non-prescription medications (including pills, patches, sprays, topical products, etc) with a focus on those for pain. Information should be collected about the dose, route, frequency, directions for use and actual use by the patient, fill quantities and dispensing patterns for each current medication and also those tried and previously discontinued. ${ }^{19}$ The BPMH should also include 
any samples, vitamins, herbal products, or traditional medicines that are used. Pharmacists should specifically ask about substance use (eg, tobacco, cannabis, alcohol, cocaine, hallucinogens, opioids, etc) as part of their history. Many people self-medicate their pain with substances alongside recreational use. Pharmacists can gather information from the patient the details about the amount, time of last substance use, how long they have used, frequency, route, source, and reasons for use.

Asking the patient to walk through their day and how medications and substances fit in can reveal what they decide to take and when, how much time is spent focusing on medication and substances, and if medications are used as prescribed. Asking the patient to bring in all of their medications from home helps to reconcile the medication list and provide an opportunity to do pill counts or remove unused medications from the home.

\section{Medication Selection and Dosing}

Therapeutic alternatives available for the management of chronic pain include acetaminophen, NSAIDs, opioids, gabapentinoids, antidepressants, cannabinoids, topical analgesics/anesthetics, and muscle relaxants. Mechanisms of action amongst these medications include facilitation of descending pain inhibitory pathways, blockade of pre-and postsynaptic receptors and neurotransmitter release, and drug targets at the level of the peripheral nociceptor. ${ }^{20}$ The choice of medication should be guided by evidence for their use in specific pain diagnoses (eg, fibromyalgia, trigeminal neuralgia), as well as guidelines based on the type of pain (eg, neuropathic) or the medication class (eg, opioids). ${ }^{21,22}$ Medication selection must be individualized, with consideration of patients' past trials and preferences. Figure 1 outlines considerations for medication selection and dosing.Figure 2

Medications with significant risks, such as opioids or cannabis, should be titrated or tapered to the lowest effective dose. Other medications must also be titrated gradually with the aim for a trial at a therapeutic dose (eg, antidepressants and gabapentinoids require gradual titration to ensure tolerability but should achieve therapeutic doses for 4-6 weeks to assess effectiveness). Pharmacists routinely provide naloxone kits to patients who use opioids and provide education to them and their families on how to use in the event of an overdose.

\section{Drug Interactions}

Pharmacists have a responsibility to identify and address drug interactions. Polypharmacy is common in chronic

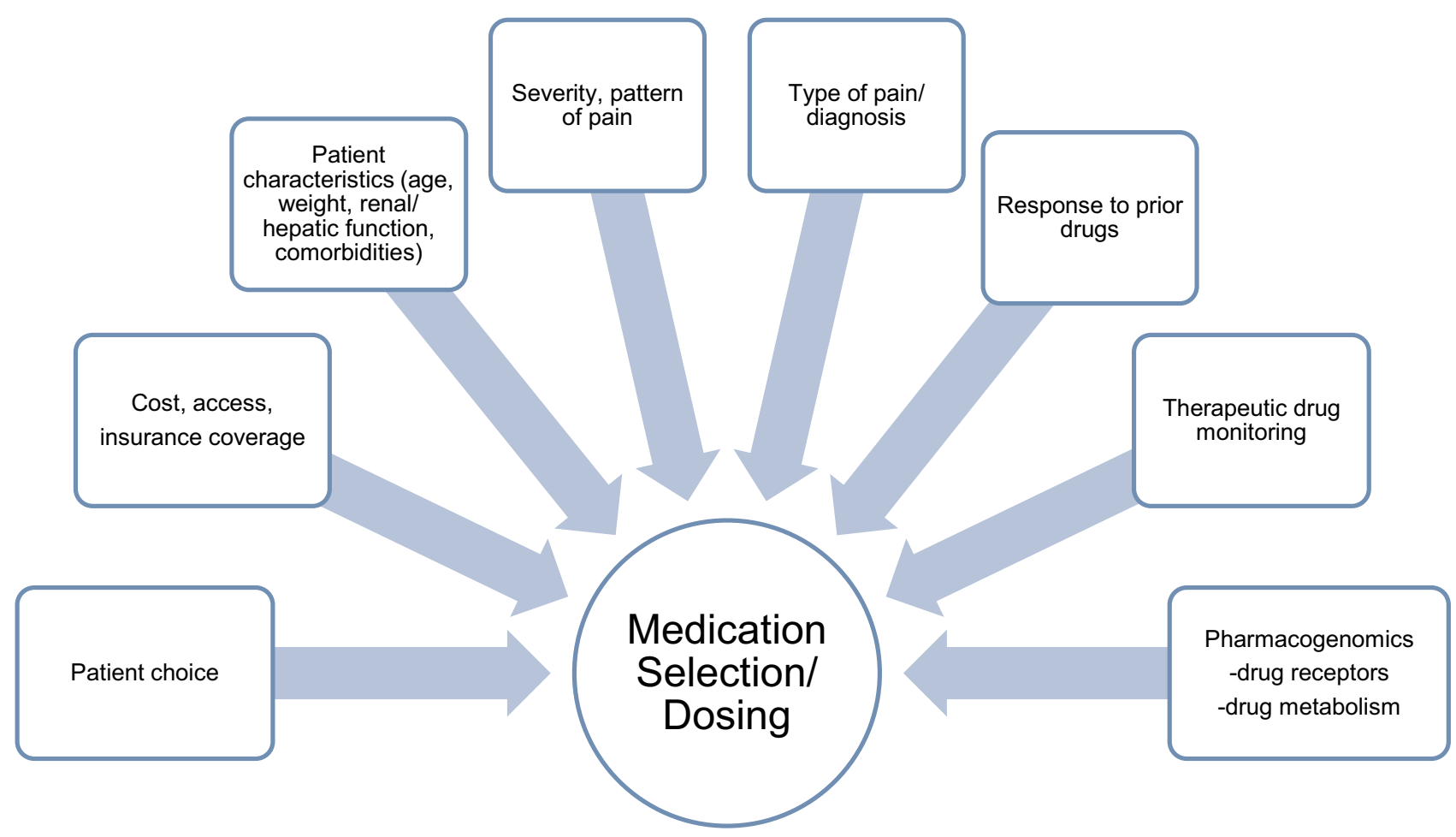

Figure I Patient factors for medication selection and dosing. 
pain management and may result in both pharmacokinetic and pharmacodynamic drug interactions. Pharmacokinetic interactions may impact the levels of medications when inhibitors of interacting enzymes are given concurrently (eg, co-administration of methadone or buprenorphine with potent CYP2D6 inhibitors can increase the serum concentrations resulting in excessive sedation and risk of overdose). ${ }^{23}$ Pharmacodynamic drug interactions between medications commonly prescribed for pain and related comorbidities may increase risk of respiratory sedation and death (eg, benzodiazepines, gabapentinoids, opioids), ${ }^{24,25}$ serotonin syndrome (eg, antidepressants, certain opioids, triptans), ${ }^{26}$ excessive anticholinergic effects (eg, tricyclic antidepressants, opioids, muscle relaxants $)^{27}$ or QT interval prolongation (eg, methadone, antidepressants, NSAIDs, muscle relaxants). ${ }^{28}$ Patients may be seeing both primary care teams and specialists to manage their physical and mental health conditions, with the pharmacist and the pharmacy as the common convergence point. As such, pharmacists are essential in assessing the medication regimen in its totality.

\section{Monitoring}

Patients living with chronic pain benefit from frequent follow-up and monitoring. Pharmacists can utilize standardized tools, such as the Brief Pain Inventory (BPI) ${ }^{29}$ to help track patients' progress over time not only with improvements in pain intensity, but also functional interference. The BPI includes a body pain diagram which may be helpful over time to identify a pattern of central sensitization (ie, a potential consequence of long-term opioid use). ${ }^{30}$

Medication dosing and safety monitoring require ongoing follow-up. In the case of opioids, calculating a morphine equivalent dose (MED) is a critical first step to compare the patient's current regimen with guidelinebased dose recommendations. In Canada, a threshold dose of $90 \mathrm{mg}$ MED is recommended for patients with chronic non-cancer pain beginning opioid therapy. Above this dose, the risks outweigh the benefits for most patients. ${ }^{22}$

Patients should be asked about adherence. A common misperception is that all medications should be taken "as needed" or sparingly, when many need to reach therapeutic steady state to be effective (eg, antidepressants). Patients receiving medications with higher abuse liability (eg, opioids, gabapentinoids) should receive additional support and be regularly screened for problematic medication-taking behaviors. Pharmacists can do this respectfully by asking the patient about how they are taking their medications, and reconcile this with data from prescription drug monitoring programs including fill history and alerts (eg, duplicate drug, double doctoring). Pharmacists can also ask about changes in route of consumption (eg, tampering, crushing, chewing oral tablets, sucking or smoking transdermal patches) and sources (eg, accessed from friends, family or "the street"). Pharmacists in various settings can also administer urine drug screening as a universal precaution to improve patient safety. ${ }^{31}$

Patients using opioids should also be screened routinely for medical complications such as sleep apnea, hypogonadism, sedation, opioid use disorder, depression, sleep interference, and constipation. Pharmacists can lead this screening in the community, with the support of brief questionnaires, such as the Routine Opioid Outcome Monitoring Tool, ${ }^{32}$ the Cannabis Use Disorder Identification Test - Revised (CUDIT-R), ${ }^{33}$ or the CAGE-AID questionnaire to screen for alcohol and substance use problems. ${ }^{34}$ If problematic use is identified, it is an opportunity for structured brief interventions, referrals and support to access care in the context of their pain treatment (eg, with methadone or buprenorphine/ naloxone).

\section{Deprescribing and Discontinuation}

Pharmacists also have a key role in identifying medications that should be discontinued. This may be due to lack of indication or effect, intolerable side effects, or known risks to the patient from drug or disease interactions. They can guide the design of a plan for medication tapering and discontinuation, in collaboration with the patient and prescriber. Many medications used for pain may cause withdrawal and discontinuation symptoms if doses are reduced too quickly or the medication is discontinued abruptly (eg, SNRIs, opioids). This can be very uncomfortable, distressing and discouraging to the patient. Tools are available, in particular for opioid tapering and discontinuation, to support pharmacists in co-creating a deprescribing plan with the patient that will work for them. ${ }^{22,35}$ Pharmacists can also support patients in behavior change, including reduction of substance use. Tobacco is one example where its use has independent implications for people living with pain as smoking increases pain intensity, and leads to poorer functional outcomes and greater long-term disability. ${ }^{36,37}$ 
- A pharmacist completed a medication review with Mr. S. They agreed that oxycodone was not effective for his low back pain, and there is risk of respiratory depression with the use of opioids in combination with benzodiazepines, central nervous system depressants such as baclofen, and sedating medications. The pharmacist recommends to taper and discontinue oxycodone, and provides him with a detailed plan. The pharmacist prescribes Mr. S a trial of duloxetine instead. This may also be helpful for his anxiety, and may replace his citalopram in time. At future visits, the pharmacist will plan with $\mathrm{Mr}$. S to discontinue his baclofen and benzodiazepine to decrease risks related to falls and sedation.

\section{Role of Pharmacists in Patients with Chronic Pain and Opioid Use Disorder}

For the subset of patients living with chronic pain and an opioid use disorder, opioid agonist treatment, such as buprenorphine-naloxone or methadone, is often prescribed with the same structure as an opioid use disorder treatment (eg, with observed doses).

Pharmacists care for people with opioid use disorder by providing: observed and take-home doses, monitoring for missed doses, adverse effects (eg, constipation, dry mouth), and toxicity (eg, intoxication or slurred speech), and assistance in their management, and communication of important clinical issues to the treatment team.

Pharmacists have up to 10 times more interactions with patients than their primary care providers for the management of chronic conditions, including pain. ${ }^{38}$ For patients prescribed opioids or medications for opioid use disorder, pharmacists may see these patients as frequently as daily for dispensing of medication and monitoring. These nonjudgmental and respectful regular short interactions with patients provide a tremendous opportunity to develop a therapeutic relationship and have positive impact on patient outcomes and quality of life.

\section{Role in Pain Education and Self-Management}

- Mr. S has never felt that people believe that he has pain. He feels like a burden to his family, and he does not want to tell them about how bad his pain gets because he would be complaining all the time. He is worried that his pain is getting worse, that soon he will not be able to stand it. He wishes he could do some of the things he used to enjoy like fishing, but he is stuck with his pain and just focuses on what will get him through the day.

Chronic pain self-management is the ability of patients to adapt strategies to better manage their symptoms, treatments, and the physical and psychological challenges of their pain experience. Self-management incorporates the physical, psychological and pharmacological strategies to cope with pain. Clinical trials have shown that teaching patients about the neurophysiology and neurobiology of pain reduces pain, catastrophizing, healthcare utilization and improves function. ${ }^{39-41}$ This involves discussing biological education contextualized within a biopsychosocial framework. ${ }^{42}$ Psychological therapies such as cognitive-behavioral therapy (CBT) address unhelpful emotions and maladaptive pain behaviors and when combined with physical therapy that promotes mobility and physical fitness, patients' function and ability to complete daily activities improve. ${ }^{43}$ Other psychological selfmanagement strategies that have been adapted in pain management include mindfulness-based stress reduction (MBSR) or Acceptance and commitment therapy (ACT).

The goal of caring for patients with chronic pain is not the elimination of pain, but a reduction in pain and improvement of quality of life and function. Pharmacists are well positioned to leverage their skill set to collaborate with patients developing realistic individualized treatment plans based on comorbid conditions, treatment expectations, and goals.

Pharmacists can be involved in delivering pain education and promoting self-management strategies that reduce overreliance on medication. Through their frequent interaction with patients, pharmacists can suggest the need for further referral to other health or wellness care providers if they notice barriers that negatively impact patient treatment outcomes.

- A pharmacist engaged Mr. S to discuss his pain, but also review his goals related to pain management. Treatment was aligned to help him meet his goals and optimize his function and quality of life. Pain education was helpful to set expectations related to chronic pain and the effectiveness of his medications, and also to review his pain coping skills. The pharmacist supported Mr. S with recommendations for sleep hygiene and encouraged appropriate exercise. During their discussion, the pharmacist helped Mr. S find the words to communicate his goals with his family. If 
additional support is needed, the pharmacist may refer him for additional counselling or therapy.

\section{Role as a Member of the Interprofessional Pain Care Team}

- Mr. S experiences a traumatic fall and is admitted to the hospital for a fractured wrist, requiring surgery. When he is discharged from hospital, he is given a prescription and instructions for follow-up with a physical therapist in the community. He will also be followed up by his surgeon, his family doctor and a pain specialist. He is provided education before he goes home by an inpatient pharmacist, and fills his prescription at his usual outpatient pharmacy. He is very overwhelmed, repeating his story multiple times, and it feels like none of his healthcare providers talk to each other.

Patient-centered care, the core of pain management, is defined as

providing care that is respectful of and responsive to individual patient preferences, needs and values and ensuring that patient values guide all clinical decisions. ${ }^{44}$

Effective dialogue using a motivational approach is essential in developing rapport with the patient to empower and engage those with pain to make medication and behavioral changes effectively. Partnering with patients by supporting individual autonomy in their healthcare decisions, enables patients to better manage complex chronic conditions because they understand their care plan, and feel comfortable communicating their needs. ${ }^{45}$

Across the continuum of healthcare, there are many opportunities where pharmacists can support the interprofessional pain care team, illustrated in Figure 2. These settings include community pharmacies, primary care teams, inpatient acute care and rehabilitation settings, long-term care, and also specialty ambulatory pain clinics. Pharmacists can screen, monitor and make treatment recommendations to the interprofessional team in all of these settings. Across transitions, medication reconciliation and medication education can be key to support safer medication use. In some regions or settings, pharmacists may have the ability to prescribe or adjust medications independently. Because of the geographical limitations to accessing specialty pain clinics that are located in urban academic settings, ${ }^{2}$ pharmacists can significantly support access to pain services particularly in rural, remote and reserve communities where the need is greatest.

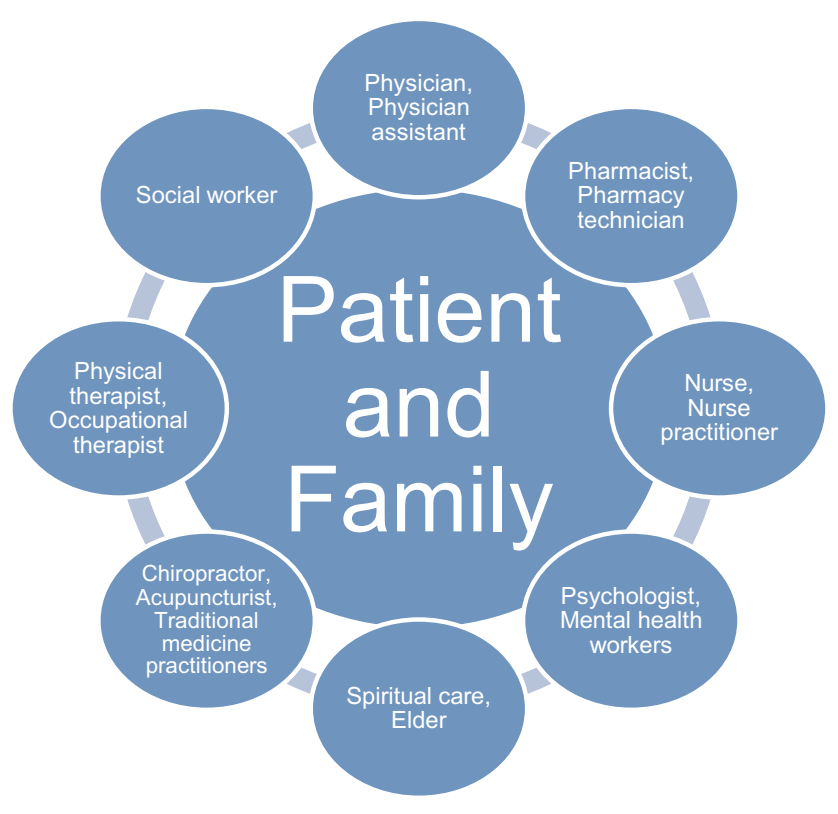

Figure 2 Interprofessional teams support pain care.

- Mr. S's pharmacist performed a discharge medication reconciliation for him and included him in medication teaching prior to his discharge from hospital. To promote seamless care, his discharge prescriptions and discharge summary were faxed to his community pharmacy. His community pharmacist also reviewed medications with him and helped him to prepare for follow-up appointments with questions for his care teams, and provided him with up to date medication lists to bring.

\section{Role to Decrease Stigma, Be Culturally Responsive, and Mitigate the Impact of Inequities}

- Mr. S feels as though his doctor and his pharmacist and the staff at the hospital treat him differently because he has chronic pain and even more so, ${ }^{46,47}$ because he is Black. He has heard whispers behind his back, that he is a "frequent flyer" and a "drug seeker". He is often dismissed, and he feels that people are focused more on telling him instead of listening to him. He is really uncomfortable and often asks for the opioid medication that he uses to be delivered so he can avoid talking to people about it.

Chronic pain disproportionately impacts women, children, Indigenous Peoples, older adults, veterans, and people who use substances. ${ }^{2}$ These groups are more likely to experience social inequities and discrimination, which influence how 
pain is experienced. ${ }^{2}$ Pharmacists must recognize that people living with chronic pain often experience stigma, which may be compounded if they also have a mental health disorder, are part of another marginalized group (eg, part of a racial or sexual minority) and/or use opioids or other substances.

Multiple forms of racism and implicit bias exist in healthcare, with significant effects on how pain is assessed and managed in Black, Indigenous and People of Color (BIPOC). ${ }^{48,49}$ Research conducted by Hoffman et al in 2016 revealed that false beliefs about biological differences persisted among white laypersons and medical trainees, leading to bias in pain perception and treatment recommendations. ${ }^{50}$ Pharmacists have a responsibility to recognize their implicit bias and create an inclusive practice for providing culturally safe care for diverse populations. Box 1 outlines five principles for pharmacists to create a culturally safe practice for diverse populations.

Box I Five Principles for Pharmacists to Create a Culturally Safe Practice for Diverse Populations

I. Practice cultural humility and create culturally safe care for diverse populations Identify and unlearn implicit bias, discrimination and stereotypes. ${ }^{46}$ Respect autonomy and acknowledge that culture-based coping strategies can reduce pain intensity and the individual's perceived impact of the pain. ${ }^{53}$ Respect the role spirituality has on health and healing.

2. Individualize care Invite the patient to tell the story of their pain. Do not assume to know or understand the situation. Avoid grouping people under labels. Employ shared decision making, and strengthsbased nurturing. ${ }^{45}$

3. Recognize that family and community are extensions of the patient

Ask people about their families to connect with them. Some patients may have chosen family, rather than biological families as key supports. Respect community-specific teaching and ceremonial protocols. $^{45}$

4. Reduce stigma and barriers to access care Language matters: avoid jargon, use person-first language. ${ }^{54}$ Employ "universal precautions" for screening and monitoring of long-term opioid use as part of routine care (eg opioid treatment agreements, urine drug testing, periodic reassessment of pain diagnosis and substance use disorder comorbidities). ${ }^{22}$ Remove barriers to care for people with substance use disorders, and offer harm reduction products and services (eg opioid agonist therapy, naloxone kits, clean needles and needle exchange).

5. Provide trauma-informed care ${ }^{45,55}$

There is an association between a history of adverse childhood experiences, trauma and chronic pain. Delivery of the five principles of trauma informed care is essential for pharmacists given their breadth of practice and connection to community.
It is important to note that the COVID-19 pandemic has illuminated health disparities in Canada and countries around the world. Responses to the pandemic have fragmented care for people living with pain, and have had significant impacts on mental health and the experience of pain, ${ }^{51,52}$ reinforcing a call to action for pharmacists to reduce stigma and support people living with pain when they may need it most.

- The pharmacist is aware of the stigma and racism that Mr. S is describing within the health care system. They strive to create an environment that is welcoming, non-judgmental, and culturally responsive, and ensure that they and their team use person-first language and avoid stigmatizing and hurtful terms. Mr. S was invited to include his family in his treatment planning. Spirituality was also included as part of a holistic treatment plan, as it was meaningful to Mr. S. The pharmacist took time to discuss with Mr. S what information can be shared within the circle of care, which built trust that his confidentiality will be maintained. To advocate for Mr. S, the pharmacist reached out to the hospital staff to discuss his concerns.

\section{Conclusion}

Pharmacists have a responsibility to collaborate with patients and ensure medications are optimized and safe as part of an overall approach to management of chronic pain and comorbidities. They often have well-developed therapeutic relationships that facilitate adoption of roles to promote self-management, provide pain education, collaborate with the interprofessional team and reduce stigma to support the person living with pain. The need for these roles in the various practice settings reinforce the importance of pain education for pharmacy students, and continuing education for practicing pharmacy professionals. Pharmacists are accessible in the community and should decrease barriers for patients in need by providing evidence-based pharmacotherapies for pain management, and opioid agonist therapy for those with an opioid use disorder. Naloxone kits and other harm reduction services should be standard care for patients using opioids long term. Pharmacists can make a positive contribution to the overall care of patients with chronic pain, supporting them to reach their individualized goals and improve their lives. 
To support the broader adoption of structured, evidence informed models for pharmacist-led services for patients living with pain, policy changes are needed to decrease scope limitations and improve access. Education for all pharmacists is needed about chronic pain and the accompanying stigma to increase readiness to support these models. Further research is needed, in particular in the community setting to demonstrate value and define helpful resources and tools, ultimately to support scaling up pharmacist services for patients living in pain in the most accessible setting.

\section{Disclosure}

The authors report no conflicts of interest in this work.

\section{References}

1. Goldberg DS, McGee SJ. Pain as a global public health priority. $B M C$ Public Health. 2011;11(1):770. doi:10.1186/1471-2458-11-770

2. Canadian Pain Task Force. Chronic pain in Canada: laying a foundation for action. Health Canada; 2019. Available from: https://www.canada.ca/content/dam/hc-sc/documents/corporate/ about-health-canada/public-engagement/external-advisory-bodies/ canadian-pain-task-force/report-2019/canadian-pain-task-force-June2019-report-en.pdf. Accessed April 2, 2021.

3. Loeser JD. Pain and suffering. Clin J Pain. 2000;16(2 Suppl):S2-S6. doi:10.1097/00002508-200006001-00002

4. Nicol AL, Sieberg CB, Clauw DJ, Hassett AL, Moser SE, Brummett CM. The association between a history of lifetime traumatic events and pain severity, physical function, and affective distress in patients with chronic pain. J Pain. 2016;17(12):1334-1348. doi:10.1016/j.jpain.2016.09.003

5. Katz L, Patterson L, Zacharias R. Evaluation of an interdisciplinary chronic pain program and predictors of readiness for change. Can J Pain. 2019;3(1):70-78. doi:10.1080/24740527.2019.1582296

6. Marra CA, Grubisic M, Cibere J, et al. Cost-utility analysis of a multidisciplinary strategy to manage osteoarthritis of the knee: economic evaluation of a cluster randomized controlled trial study. Arthritis Care Res (Hoboken). 2014;66(6):810-816. doi:10.1002/acr.22232

7. Bruhn H, Bond CM, Elliott AM, et al. Pharmacist-led management of chronic pain in primary care: results from a randomised controlled exploratory trial. BMJ Open. 2013;3(4):e002361. doi:10.1136/bmjopen-2012-002361

8. Canadian Pain Task Force. Working together to better understand, prevent and, manage chronic pain: what we heard. Health Canada; 2020. Available from: https://www.canada.ca/content/dam/hc-sc/docu ments/corporate/about-health-canada/public-engagement/externaladvisory-bodies/canadian-pain-task-force/report-2020-rapport/report2020.pdf. Accessed April 2, 2021.

9. Thapa P, Lee SWH, Kc B, Dujaili JA, Mohamed Ibrahim MI, Gyawali S. Pharmacist-led interventionon chronic pain management: A systematic review and meta-analysis. Br J Clin Pharmacol. Jan 24 2021; doi: $10.1111 /$ bcp 14745

10. Dole EJ, Murawski MM, Adolphe AB, Aragon FD, Hochstadt B. Provision of pain management by a pharmacist with prescribing authority. Am J Health Syst Pharm. 2007;64(1):85-89. doi:10.2146/ ajhp060056

11. Wiedemer NL, Harden PS, Arndt IO, Gallagher RM. The opioid renewal clinic: a primary care, managed approach to opioid therapy in chronic pain patients at risk for substance abuse. Pain Med. 2007;8 (7):573-584. doi:10.1111/j.1526-4637.2006.00254.x
12. Jacobs SC, Son EK, Tat C, Chiao P, Dulay M, Ludwig A. Implementing an opioid risk assessment telephone clinic: outcomes from a pharmacist-led initiative in a large Veterans Health Administration primary care clinic, December 15, 2014-March 31, 2015. Subst Abus. 2016;37(1):15-19. doi:10.1080/ 08897077.2015.1129527

13. Strand MA, Eukel H, Burck S. Moving opioid misuse prevention upstream: a pilot study of community pharmacists screening for opioid misuse risk. Res Social Adm Pharm. 2019;15(8):1032-1036. doi:10.1016/j.sapharm.2018.07.011

14. Phueanpinit P, Pongwecharak J, Krska J, Jarernsiripornkul N. Evaluation of community pharmacists' roles in screening and communication of risks about non-steroidal anti-inflammatory drugs in Thailand. Prim Health Care Res Dev. 2018;19(6):598-604. doi:10.1017/s1463423618000142

15. Tabeefar H, Chang F, Cooke M, Patel T. Community pharmacists and chronic pain: a qualitative study of experience, perception, and challenges. Can J Pain. 2020;4(3):29-39. doi:10.1080/ 24740527.2020.1749516

16. Patel T, Chang F, Mohammed HT, et al. Knowledge, perceptions and attitudes toward chronic pain and its management: a cross-sectional survey of frontline pharmacists in Ontario, Canada. PLoS One. 2016;11(6): e0157151. doi:10.1371/journal.pone.0157151

17. Hartung DM, Hall J, Haverly SN, et al. Pharmacists' role in opioid safety: a focus group investigation. Pain Med. 2018;19 (9):1799-1806. doi:10.1093/pm/pnx139

18. Giannitrapani KF, Glassman PA, Vang D, et al. Expanding the role of clinical pharmacists on interdisciplinary primary care teams for chronic pain and opioid management. BMC Fam Pract. 2018;19 (1):107. doi:10.1186/s12875-018-0783-9

19. Safer Healthcare Now. Best possible medication history interview guide. Available from: https://www.ismp-canada.org/download/ MedRec/SHN_medcard_09_EN.pdf. Accessed April 2, 2021.

20. Finnerup NB. Nonnarcotic methods of pain management. $N$ Engl J Med. 2019;380(25):2440-2448. doi:10.1056/NEJMra1807061

21. Moulin D, Boulanger A, Clark AJ, et al. Pharmacological management of chronic neuropathic pain: revised consensus statement from the Canadian Pain Society. Pain Res Manag. 2014;19(6):328-335. doi:10.1155/2014/754693

22. Busse JW, Craigie S, Juurlink DN, et al. Guideline for opioid therapy and chronic noncancer pain. CMAJ. 2017;189(18):E659-E666. doi:10.1503/cmaj. 170363

23. Holmquist GL. Opioid metabolism and effects of cytochrome P450. Pain Med. 2009;10(suppl_1):S20-S29. doi:10.1111/j.1526-4637.2009.00596.x

24. Gomes T, Juurlink DN, Antoniou T, Mamdani MM, Paterson JM, van den Brink W. Gabapentin, opioids, and the risk of opioid-related death: a population-based nested case-control study. PLoS Med. 2017;14(10):e1002396. doi:10.1371/journal.pmed.1002396

25. Sun EC, Dixit A, Humphreys K, Darnall BD, Baker LC, Mackey S. Association between concurrent use of prescription opioids and benzodiazepines and overdose: retrospective analysis. BMJ. 2017;356: j760. doi:10.1136/bmj.j760

26. Volpi-Abadie J, Kaye AM, Kaye AD. Serotonin syndrome. Ochsner J. 2013;13(4):533-540.

27. Haanpää ML, Gourlay GK, Kent JL, et al. Treatment considerations for patients with neuropathic pain and other medical comorbidities. Mayo Clin Proc. 2010;85(3 Suppl):S15-S25. doi:10.4065/mcp.2009.0645

28. Klivinyi C, Bornemann-Cimenti H. Pain medication and long QT syndrome. Korean J Pain.. Ann Acad Med Singapore. 2018;31 (1):3-9.doi:10.3344/kjp.2018.31.1.3

29. Cleeland CS, Ryan KM. Pain assessment: global use of the brief pain inventory. Ann Acad Med Singapore. 1994;23(2):129-138.

30. Yi P, Pryzbylkowski P. Opioid induced hyperalgesia. Pain Med. 2015;16(Suppl 1):S32-6. doi:10.1111/pme.12914 
31. Gourlay DL, Heit HA, Almahrezi A. Universal precautions in pain medicine: a rational approach to the treatment of chronic pain. Pain Med. 2005;6(2):107-112. doi:10.1111/j.1526-4637.2005.05031.x

32. Nielsen S, Picco L, Kowalski M, et al. Routine opioid outcome monitoring in community pharmacy: outcomes from an open-label single-arm implementation-effectiveness pilot study. Res Social Adm Pharm. 2020;16(12):1694-1701. doi:10.1016/j.sapharm.2020.02.009

33. Adamson SJ, Kay-Lambkin FJ, Baker AL, et al. An improved brief measure of cannabis misuse: the Cannabis Use Disorders Identification Test-Revised (CUDIT-R). Drug Alcohol Depend. 2010;110(1-2):137-143. doi:10.1016/j.drugalcdep.2010.02.017

34. Brown RL, Rounds LA. Conjoint screening questionnaires for alcohol and other drug abuse: criterion validity in a primary care practice. Wis Med J. 1995;94(3):135-140.

35. Regier L. Opioid Tapering Template. RxFiles; 2017.

36. Patterson AL, Gritzner S, Resnick MP, Dobscha SK, Turk DC, Morasco BJ. Smoking cigarettes as a coping strategy for chronic pain is associated with greater pain intensity and poorer pain-related function. J Pain. 2012;13(3):285-292. doi:10.1016/j. jpain.2011.11.008

37. Shi Y, Weingarten TN, Mantilla CB, Hooten WM, Warner DO. Smoking and pain: pathophysiology and clinical implications. Anesthesiology. 2010;113(4):977-992. doi:10.1097/ALN.0b013e3181ebdaf9

38. Tsuyuki RT, Beahm NP, Okada H, Al Hamarneh YN. Pharmacists as accessible primary health care providers: review of the evidence. Can Pharm J (Ott). 2018;1:4-5. doi:10.1177/1715163517745517

39. Van Oosterwijck J, Nijs J, Meeus M, et al. Pain neurophysiology education improves cognitions, pain thresholds, and movement performance in people with chronic whiplash: a pilot study. $J$ Rehabil Res Dev. 2011;48(1):43-58. doi:10.1682/jrrd.2009.12.0206

40. Louw A, Zimney K, O'Hotto C, Hilton S. The clinical application of teaching people about pain. Physiother Theory Pract. 2016;32 (5):385-395. doi:10.1080/09593985.2016.1194652

41. Lee H, McAuley JH, Hübscher M, Kamper SJ, Traeger AC, Moseley GL. Does changing pain-related knowledge reduce pain and improve function through changes in catastrophizing? Pain. 2016;157(4):922-930. doi:10.1097/j.pain.0000000000000472

42. Moseley GL, Butler DS. Fifteen years of explaining pain: the past, present, and future. J Pain. 2015;16(9):807-813. doi:10.1016/j. jpain.2015.05.005

43. Shaikh M, Hapidou EG. Factors involved in patients' perceptions of self-improvement after chronic pain treatment. Can J Pain. 2018;2 (1):145-157. doi:10.1080/24740527.2018.1476821
44. Institute of Medicine (US) Committee on Quality of Health Care in America. Crossing the Quality Chasm: A New Health System for the 21st Century. Washington (DC): National Academies Press (US); 2001. Executive Summary. Available from: https://www.ncbi.nlm. nih.gov/books/NBK222271

45. Bergeson SC, Dean JD. A systems approach to patient-centered care. JAMA. 2006;296(23):2848-2851. doi:10.1001/jama.296.23.2848

46. Sasakamoose J, Bellegarde T, Sutherland W, Pete S, McKayMcNabb K. Miýo-pimātisiwin developing Indigenous Cultural Responsiveness Theory (ICRT): improving indigenous health and well-being. Int Indig Policy J. 2017;8(4). doi:10.18584/iipj.2017.8.4.1

47. Edgoose JYC, Quiogue M, Sidhar K. How to identify, understand, and unlearn implicit bias in patient care. Fam Pract Manag. 2019;26 (4):29-33.

48. Wylie L, McConkey S. Insiders' insight: discrimination against indigenous peoples through the eyes of health care professionals. J Racial Ethn Health Disparities. 2019;6(1):37-45. doi:10.1007/ s40615-018-0495-9

49. Lee P, Le Saux M, Siegel R, et al. Racial and ethnic disparities in the management of acute pain in US emergency departments: meta-analysis and systematic review. Am J Emerg Med. 2019;37 (9):1770-1777. doi:10.1016/j.ajem.2019.06.014

50. Hoffman KM, Trawalter S, Axt JR, Oliver MN. Racial bias in pain assessment and treatment recommendations, and false beliefs about biological differences between blacks and whites. Proc Natl Acad Sci. 2016;113(16):4296. doi:10.1073/pnas.1516047113

51. Webster F, Connoy L, Sud A, Pinto AD, Katz J. Grappling with chronic pain and poverty during the COVID-19 pandemic. Can J Pain. 2020;4(1):125-128. doi:10.1080/24740527.2020.1766855

52. Lynch ME, Williamson OD, Banfield JC. COVID-19 impact and response by Canadian pain clinics: a national survey of adult pain clinics. Can J Pain. 2020;4(1):204-209. doi:10.1080/ 24740527.2020.1783218

53. Peacock S, Patel S. Cultural influences on pain. Reviews in Pain. 2008;1(2):6-9. doi:10.1177/204946370800100203

54. Broyles LM, Binswanger IA, Jenkins JA, et al. Confronting inadvertent stigma and pejorative language in addiction scholarship: a recognition and response. Subst Abus. 2014;35(3):217-21. doi:10.1080/ 08897077.2014.930372

55. Purkey E, Patel R. Phillips SP. Trauma-informed care: Better care for everyone. Canadian Family Physician. 2018;64(3):170-172.
Integrated Pharmacy Research and Practice

\section{Publish your work in this journal}

Integrated Pharmacy Research and Practice is an international, peerreviewed, open access, online journal, publishing original research, reports, reviews and commentaries on all areas of academic and professional pharmacy practice. This journal aims to represent the academic output of pharmacists and pharmacy practice with particular focus on integrated care. All papers are carefully peer reviewed to ensure the highest standards as well as ensuring that we are informing and stimulating pharmaceutical professionals. The manuscript management system is completely online and includes a very quick and fair peer-review system, which is all easy to use. Visit http://www.dovepress.com/testimonials.php to read real quotes from published authors. 